\title{
miR-145 suppresses epithelial-mesenchymal transition by targeting stem cells in Ewing sarcoma cells
}

\author{
Guzel Tanoglu E${ }^{1}$, Ozturk $S^{2}$ \\ Department of Molecular Biology and Genetics, Institute of Health Sciences, University of Health Sciences \\ Turkey, Istanbul, Turkey. esra.guzel@sbu.edu.tr
}

\section{ABSTRACT}

OBJECTIVES: We aimed to examine the performance of stem cell markers and epithelial-mesenchymal transition (EMT) process in miR-145 transfected EWS cells (TC71, TC106).

METHODS: EWS cells were utilized for functional analysis of mir-145. Proliferation, migration, invasion and soft agar colony assay were performed to observe the alterations in migration behavior of transfected cells. Caspase assay was used to investigate the underlying reasons of proliferative inhibition in cells in which miR-145 is overexpressed. QRT-PCR was used to determine the role of miR-145 in EMT transcription markers and mir-145 targeted genes, KLF4, SOX2 and OCT4 expression levels.

RESULTS: The miR-145 expression has been shown to be down-regulated in EWS. The miR-145 overexpression caused inhibition of proliferation and reduced migration in EWS cells through induction of apoptosis. Mir-145 suppresses EMT capacity and SOX2, KLF4 and OCT4 expression levels.

CONCLUSION: This is the first time in the literature we have shown deregulation of miR-145 inhibits EMT process by targeting stem cell properties leading to the inhibition of tumor growth and metastasis in TC71 and TC106 cells. Based on these results, we propose that miR-145, as an important regulator of SOX2, KLF4 and OCT4 carries crucial roles in EWS tumorigenesis and EMT (Tab. 1, Fig. 4, Ref. 26). Text in PDF www.elis.sk

KEY WORDS: Ewing sarcoma, mir-145, stem cell markers.

\section{Introduction}

Ewing Sarcoma (EWS) is undifferentiated malignant tumor of small round cells in children and young adults (1). Moreover, it is the second most commonly seen neoplasm of bone tumors. Approximately $85 \%$ of Ewing sarcoma cases have locally advanced or metastatic disease at diagnosis. Approximately $90 \%$ of cases with Ewing Sarcoma most commonly contain EWS gene on chromosome 22 fused to FLI1 on chromosome 11 which chimeric gene encodes an EWS-FLI1 (2). Therapeutic options which are the preferentially used to treat early and localized EWS commonly fail to give positive clinical results. It has been reported that the rate of metastasis is $20-30 \%$ in pediatric patients diagnosed with Ewing Sarcoma. Therapeutic methods for the optimal management of Ewing cancer are also inadequate due to the complex background of this cancer. Although effective new methods for

${ }^{1}$ Department of Molecular Biology and Genetics, Institute of Health Sciences, University of Health Sciences Turkey, Istanbul, Turkey, and ${ }^{2}$ Department of Internal Medicine, Division of Medical Genetics, Istanbul Medical Faculty, Istanbul University, Istanbul, Turkey.

Address for correspondence: E.Guzel Tanoglu, University of Health Sciences, Mekteb-i Tibbiye-i Şahane (Haydarpaşa) Külliyesi, Selimiye Mah. Tibbiye Cad. No:38, 34668, Uskudar, Istanbul, Turkey. Phone: +90.216 .4189616$

Acknowledgement: This study was supported by Istanbul University Scientific Research Project. (Grant Number 21573). the treatment of early and non-metastasizing Ewing Sarcoma cases are being tried, approaches for the treatment of advanced-stage cases are needed (2).

MicroRNAs (miRNA) are regulatory single-stranded noncoding small RNAs including nearly 20 nucleotides and accepted as a class of gene regulators. miRNAs suppress expression of their target genes via mRNA translational or degradation decrease as a consequence of incomplete binding to the 3 'untranslated regions (3'UTR) of mRNAs (3). The dysfunction of miRNAs has been exhibited to have a role in the development of many diseases and cancer progression (4) and their abnormal expression is evaluated in EWS cells and tumor samples. MiR-145, which is localized on the 5 th chromosome, is intergenic and the sequence of the mature form is 5'-GUCCAGUUUUCCCAGGAAUCCCUU-3. miR-145 has a tumor suppressor role and is highly conserved among species. Mir-145 is targeting multiple oncogenes regulating various biological processes such as cell cycle, proliferation, migration and apoptosis (5). It has been formerly stated that miR145 is downregulated in many tumor types such as larynx (6), colorectal (7), prostate (8), and acute myeloid leukemia (9). SOX2, KLF4, and OCT4 are cell-fate determining transcription factors that have been sustaining the self-renewal and pluripotency properties of induced pluripotent stem (iPS) and embryonic stem cells (10). It has been stated that SOX2, OCT4 and KLF4, known as stem cell markers, are targeted by mir-145 and mir-145 acting in order to maintain the efficacy of cancer stem cells (11). Many papers have linked the 
cancer stem cells phenotype to carcinoma cells undergoing epithelial to mesenchymal transition (EMT) (12). The EMT behavior is defined by a transition from immotile epithelial cells to motile mesenchymal cells, then this progression is causing promotion of cell proliferation, migration, and invasion in malignant cells. This transition is determined by a decrease in the expression of proteins that are items of the adherence junction complex and enhance cellcell contact such as E-cadherin, as well as a rise in the expression of mesenchymal markers such as vimentin, N-cadherin, and fibronectin, and several transcription mediators, including Snail, Slug/Snail2, ZEB1, ZEB2 (13).

In this paper, we aimed to investigate the biological role of mir-145 on Ewing Sarcoma cells.

\section{Methods}

Cell lines

The TC-71 and TC-106 (EWS) cell lines were purchased from 'Children's Oncology Group' (Texas). The cells were grown in IMDM (GIBCO) with L-Glutamine, $10 \% \mathrm{FCS}, 10 \mathrm{U} / \mathrm{ml}$ and penicillin-streptomycin.

\section{Cell culture and transfection}

TC-71 and TC 106 cell lines are used for functional investigations of miR-145. The cell lines, $10 \%$ Fetal Bovine Serum (FBS), $1 \%$ Penicillin, Streptomycin, Ampicillin (PSA) and LGlutamine on IMDM medium at $37{ }^{\circ} \mathrm{C}$ were cultured in an oven set. MiR-145 and non-targeting control were obtained from Sigma. miRNA transfections were accomplished using the Lipofectamine RNAiMAX Transfection Reagent (Invitrogen) according to the manufacturer's advice.

\section{Proliferation assay}

The proliferation abilities of TC71, TC106 cells transfected with either miR-145 or nontargeting miRNA either miR-145 or nontargeting miRNA were determined using MTS assay. Cells were plated in 96 well plates overnight in triplicate and the cells transfected with miR-145 or nontargeting miR using Lipofectamine RNAiMAX Transfection Reagent (Invitrogen). In order to test cell proliferation rates every 24 hours for 2 days, cell proliferation reagent, WST1 (Roche, Mannheim, Germany), was used and cells were incubated at $37^{\circ} \mathrm{C}$ for 3 hours in dark conditions. Absorbance was measured at $490 \mathrm{~nm}$ using Multiscan FC micro plate reader (Thermo, Rockford, IL).

\section{Migration and invasion assay}

The effect of miR-145 on the migration and invasion ability of TC71 and TC106 cells was assessed by BD BioCoat Matrigel invasion chambers (Becton Dickinson).

Matrigel-Tris $\mathrm{HCl}$ mixture prepared in the ratio of 1: 5 was added to the inserts as $500 \mathrm{ml}$ and incubated at $37^{\circ} \mathrm{C}$ for 4 hours. Mir-145 and control miRNA transfected cells were seeded into each chamber at a density of $3 \times 10^{5}$ cells in triplicates. The medium with FPS was placed on the bottom surface of the inserts and the cells were kept in the incubator for 24 hours to learn the migration and invasion potential. After 1 day non-invading cells were removed and the invading cells on the lower surface of the filter were fixed with $100 \%$ methanol. For the dyeing process, the mixture consisting of crystal violet $(0.01 \%)$, ethanol (10\%) and distilled water was treated for $20 \mathrm{~min}$. Cells that appear in the insert are counted and analyzed.

\section{Soft agar colony assay}

Cells transfected with miR-145 or non-targeting miR were suspended in a density of 3000 cells $/ \mathrm{ml}$ in $1.2 \%$ low melting agar $(0.36 \mathrm{~g})$ diluted in IMDM and plated on a $0.6 \%$ base agar in 6 -well plates. Fixation and staining were carried out in a mixture of $0.01 \%$ crystal violet, $10 \%$ ethanol dissolved in distilled water after 14 to 30 days in culture at $37^{\circ} \mathrm{C}$. Cells were counted with a dissecting microscope after washing 3 times with distilled water to remove background staining.

\section{Caspase assay}

EWS cells were seeded into 96-well plates to determine apoptotic response upon mir-145 transfection. At 48th and $72 \mathrm{nd} \mathrm{h}$ after transfection the cells were harvested and colorimetric caspase 3 activity assay was performed using "CaspACETM Assay System, Colorimetric (Promega)" with respect to the manufacturer's instruction. Caspase activity was determined by measuring the absorbance at $405 \mathrm{~nm}$ with an Multiscan FC micro plate reader (Thermo, Rockford USA).

\section{RNA isolation and quantitative real-time PCR}

Total RNA was extracted from cell lines with TRIzoL with respect to the manufacturer's protocol. The purities and concentrations of RNA samples were carried out by spectrophotometry using

Tab. 1. The primers used in this study.

\begin{tabular}{|c|c|c|}
\hline Gene & $5 ' \rightarrow 3^{\prime}$ & References \\
\hline Beta-actin-F & GCCTCGCCTTTGCCGATC & \multirow{2}{*}{$(25)$} \\
\hline Beta-actin-R & CCCACGATGGAGGGGAAG & \\
\hline E-Cad-F & TGCCCAGAAAATGAAAAAGG & \multirow{2}{*}{ (26) } \\
\hline$E$-Cad-R & GTGTATGTGGCAATGCGTTC & \\
\hline$N-C a d-F$ & ACAGTGGCCACCTACAAAGG & \multirow{2}{*}{ (26) } \\
\hline$N$-Cad-R & CCGAGATGGGGTTGATAATG & \\
\hline SNAIL1-F & AAGATGCACATCCGAAGCC & \multirow{2}{*}{ (26) } \\
\hline SNAIL1-R & CGCAGGTTGGAGCGGTCAGC & \\
\hline SNAIL2-F & ATACCACAACCAGAGATCCTCA & \multirow{2}{*}{$(26)$} \\
\hline SNAIL2-R & GACTCACTCGCCCCAAAGATG & \\
\hline$Z E B 1-F$ & GATGATGAATGCGAGTCAGATGC & \multirow{2}{*}{$(26)$} \\
\hline$Z E B 1-R$ & ACAGCAGTGTCTTGTTGTTGTAG & \\
\hline ZEB2-F & AACAACGAGATTCTACAAGCCTC & \multirow{2}{*}{$(26)$} \\
\hline ZEB2-R & TCGCGTTCCTCCAGTTTTCTT & \\
\hline Fibronectin-F & TCCCTCGGAACATCAGAAAC & \multirow{2}{*}{ (26) } \\
\hline Fibronectin- $R$ & CAGTGGGAGACCTCGAGAAG & \\
\hline Vimentin-F & GAGAACTTTGCCGTTGAAGC & \multirow{2}{*}{$(26)$} \\
\hline Vimentin- $R$ & GCTTCCTGTAGGTGGCAATC & \\
\hline$S O X 2-F$ & CTCCGGGACATGATCAGC & \multirow{2}{*}{$(25)$} \\
\hline$S O X 2-R$ & GGTAGTGCTGGGACATGTG & \\
\hline$K L F 4-F$ & CCСАATTAСССАТССТТССТG & \multirow{2}{*}{ (25) } \\
\hline$K L F 4-R$ & GTCTTCCCCTCTTTGGCTTG & \\
\hline OCT4-F & CTGGAGAAGGAGAAGCTGGA & \multirow{2}{*}{$(25)$} \\
\hline OCT4-R & CAAATTGCTCGAGTTCTTTCTG & \\
\hline
\end{tabular}


A
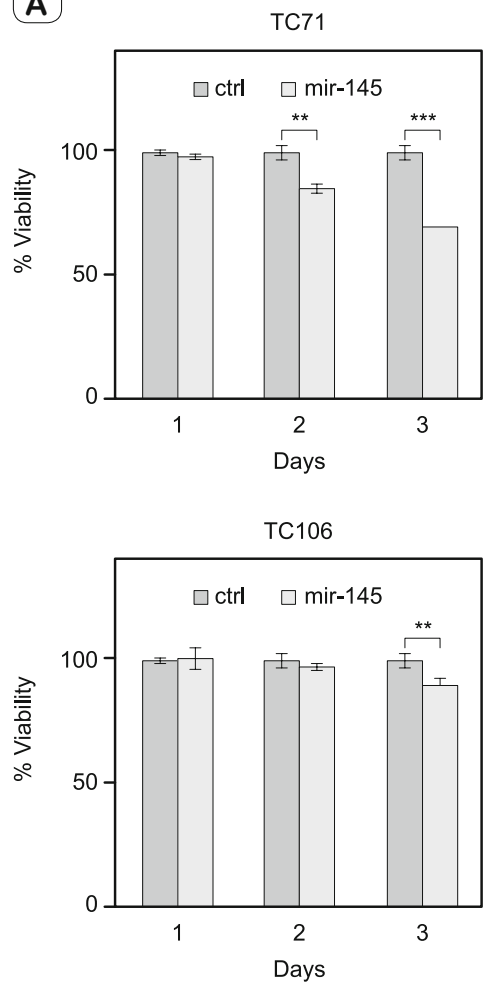

(D)
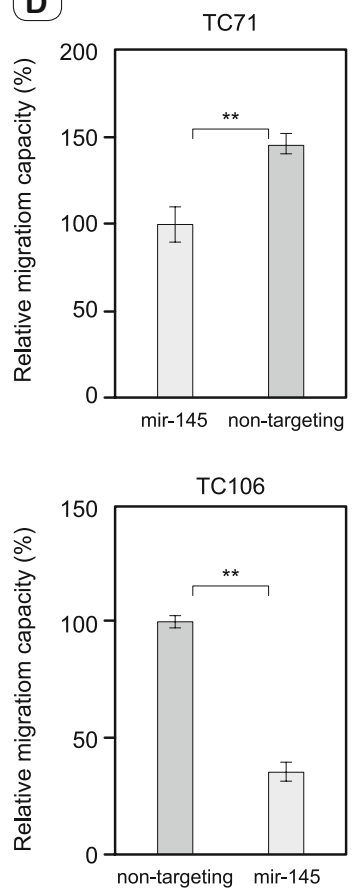

B

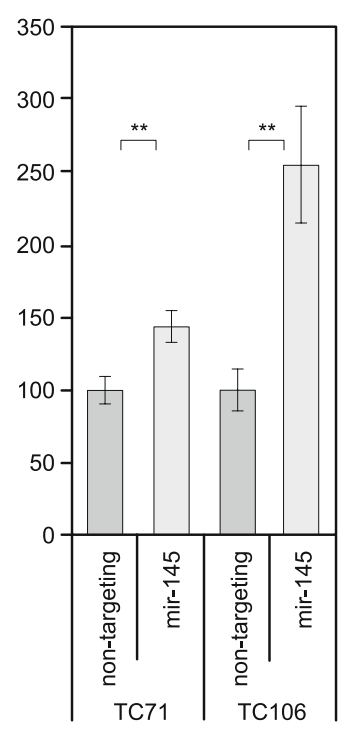

C
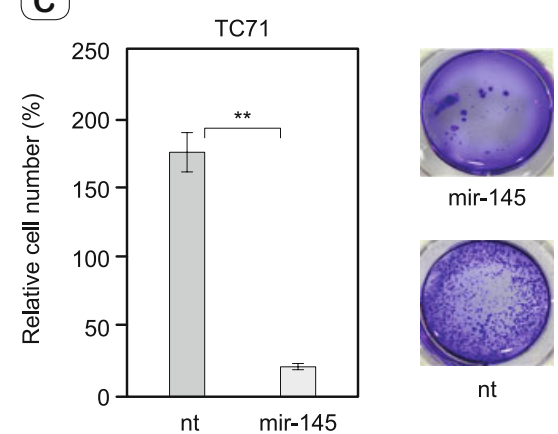

mir-145

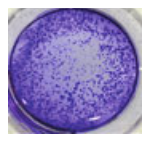

nt
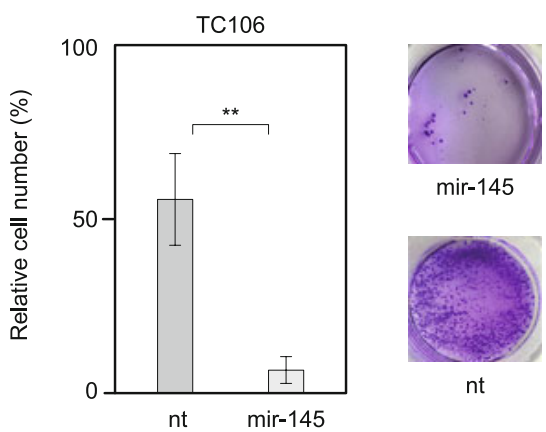

mir-145

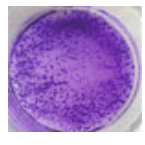

$\mathrm{nt}$

E
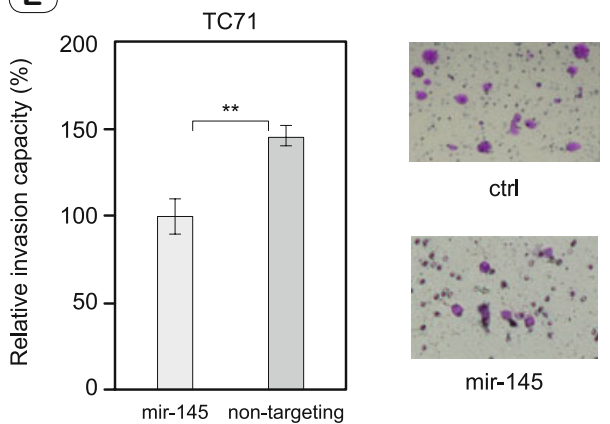

ctrl

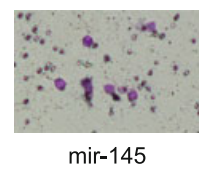

mir-145

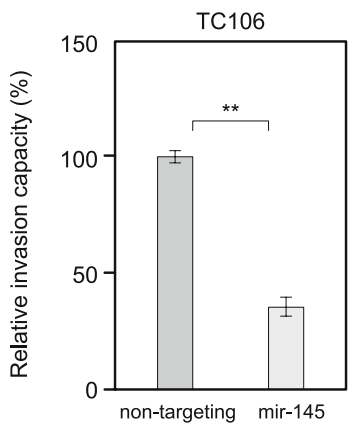

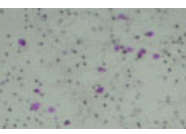

ctrl

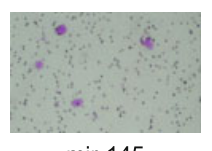

mir-145

Fig. 1. Functional impact of miR-145 overexpression in EWS cells. (A) Treatment with miR-145 inhibited cell proliferation (B) apoptosis, and (C) colony capacity in (D) migration and (E) invasion assay EWS cell lines TC71 and TC106. * $p<0.01, * * p<0.01$, $* * * p<0.001$ significant difference between two compared groups. 
NanoDrop ND-2000c (Thermo Fisher Scientific, Inc., Wilmington, $\mathrm{DE})$. To validate the differential expressions of miR-145 in cell lines were determined. First strand DNA (cDNA) synthesis was performed $30 \mathrm{ng}$ of total RNA from every sample using mir-145, and used as a control RNU43 primers purchased from Applied Biosystems and "TaqMan MicroRNA Reverse Transcription Kit" compatible with the manufacturer's instruction (Applied Biosystems, Foster City, CA). Quantitative RT-PCR was accomplished using TaqMan Universal Master Mix, Applied Biosystems (Foster City, CA) and microRNA specific probes were obtained from Applied Biosystems (Denmark) in a Roche LightCycler480-II realtime thermal cycler (Roche, Switzerland).

\section{Syber green quantitative real-time PCR}

The SYBR Green qRT-PCR reaction for detection and quantification of targeted miRNA genes was performed with $1 \mu \mathrm{g}$ of RNA was reverse transcribed using Transcriptor High Fidelity cDNA synthesis kit (Roche, Switzerland) with respect to the manufacturer's instructions. The relative expression levels of SOX2, KLF4, OCT4 were tested by qPCR on a Light Cycler 480 utilizing Light Cycler 480 SYBR Green I Master (Roche). A SYBR Green qRT-PCR reaction for GAPDH was used as an internal control and qRT-PCR reactions were carried out in triplicate for each specimen.

SYBR Green qRT-PCR was performed at 1 cycle of $95^{\circ} \mathrm{C}$ for 5 min followed by 40 cycles of $95{ }^{\circ} \mathrm{C}$ for 10 seconds, $60{ }^{\circ} \mathrm{C}$ for 20 seconds and $72{ }^{\circ} \mathrm{C}$ for 25 seconds. Melting curve analysis was performed at temperatures between $65^{\circ}$ and $95^{\circ}$. Primer sequences used for qRT-PCR are given in Table 1.

SOX2, OCT4 and KLF4 expression levels, were analyzed with qRT-PCR in TC-71 and TC-106 cell lines transfected with mir-145. Non-target miRNAs transfected into TC-71 and TC-106 were used as controls. It showed that the optimum annealing temperature for SOX2 primers was $60{ }^{\circ} \mathrm{C}$. Gene expression change values that were verified by qRT-PZR were determined by deltadelta-Ct analysis.

\section{Statistical analysis}

All data were tested using two-sided Student's t-test. Data were plotted as mean \pm standard deviation to evaluate the significance of differences between the expression levels of the tested genes in EWS cell lines.

\section{Results}

In order to determine the effectiveness of miR-145 mimic transfection in cells we have confirmed mir-145 expression levels in TC-106 and TC-71 cells by qRT-PCR. 24 hours after transfection, mir-145 expression changes reached up to $\sim 20$ fold higher in mir-145 transfected cell lines compared to non-target miRNA transfected cells.

In order to evaluate the role of mir-145 in tumorigenesis, we used the functional assays in EWS cells. The proliferation assay exhibited that overexpression of miR-145 in the EWS cells brought about a significant decline in the proliferation rate. The prolifera- tion rate of EWS cells significantly differed at 24 and 48 hours after miR-145 overexpression (Fig. 1A). It was determined that mir-145 transfected TC-106 and TC-71 cells decreased compared to nt-transfected cells. In TC71 and TC106 cells, miR-145 overexpression decreased migration by approximately $50 \%$, invasion by up to approximately $75 \%$ (Fig. 1D-E).

Caspase- 3 levels were measured to investigate the underlying causes of proliferation reduction after EWS cells were transfected with mir-145, we performed caspase-3 activity assay. Caspase-3 activity rose as a result of the increase of apoptotic cells. The caspase- 3 activity assay showed that the increased expression of miR-145 in EWS cells caused an approximately 1.5 and 2.5-fold increase in the caspase-3 activity compared with non-targeting miRNA cells, respectively (Fig. 1B). Soft agar colony assay was also accomplished to evaluate the colony-forming potential of mir-145 cells transfected into TC-71 and TC-106. In TC-71 and TC-106 cells, fewer colonies were observed in mir-145 transfected cells than in control miRNA. The results were calculated by taking the average of three different areas with three replicates (Fig. 1C).

\section{miR-145 inhibits the EMT}

We investigated the SOX2, KLF4, OCT4 expression levels of EMT metastasis markers in cells transfected with mir-145. The relative expression level of EMT markers in mir-145 transfected cell lines is shown in figures $3 \mathrm{a}$ and $3 \mathrm{~b}$. Overall, the results exactly exhibited that miR-145 overexpression in EWS cells decreases their migratory potentials.

Additionally, mir-145 target SOX2, KLF4, OCT4 is also involved in EMT regulation. To determine this possibility, we transfected TC71 and TC106 cells with miR-145 and then investigated the expression of the epithelial markers (E-cadherin and $N$-cadherin, as well as the mesenchymal marker Snail1, Snail2, Vimentin, Fibronectin, ZEB1, ZEB2) using realtime PCR. We found that E-cadherin levels were rised when miR-145 were overexpressed in TC71 (Fig. 3A) and TC106 (Fig. 3B) cells. Other EMT markers showed that miR-145 overexpression caused decline in the expression of $\mathrm{N}$-cadherin and a significant decrease in the expression of ZEB1, ZEB2, Snail1, Snail2, Vimentin and Fibronectin in both EWS cells (Figs 2A,B). Zeb2 and Snaill have shown a slightly decreased expression levels in mir-145 transfected TC71 cells as shown in Figure 3A. These results showed that overexpression of miR-145 inhibits EWS cell migration and EMT.

\section{Evaluation of Mir-145 transfected cells in terms of stem cell markers}

The expression level of SOX2, KLF4 and OCT4, the target genes of mir-145, was determined by qRT-PCR in mir-145 transfected TC-71 and TC-106 cells, and expression levels of stem cell markers were significantly suppressed in transfected cells (Fig. 3A). As a result of the decreased mir-145 level, increased expressions of SOX2, KLF4 and OCT4 demonstrated that mir-145 plays a pivotal role in the formation of Ewing sarcoma (Fig 3B). 
(A)
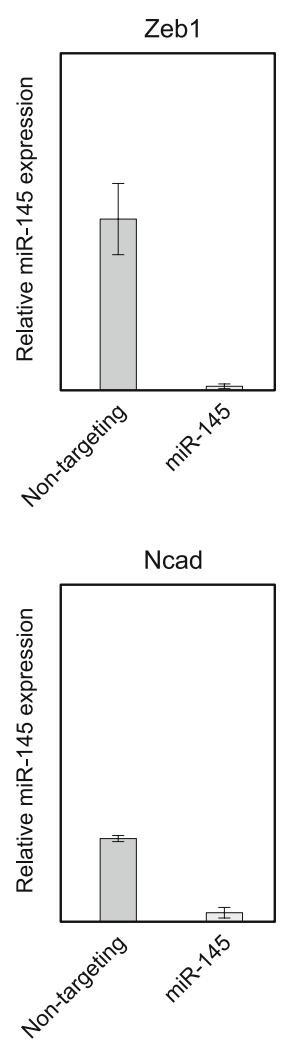

(B)
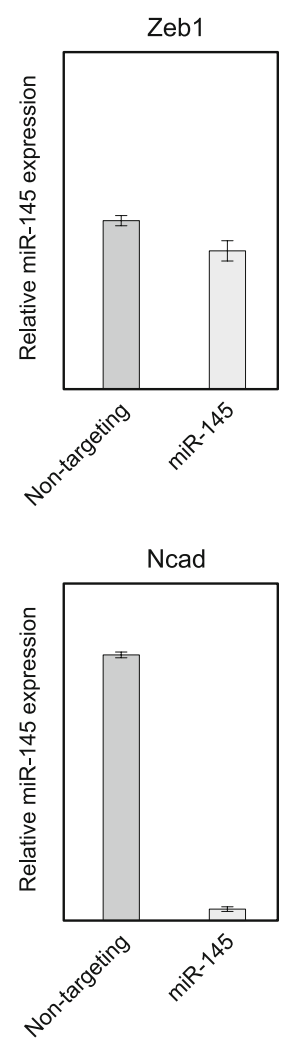
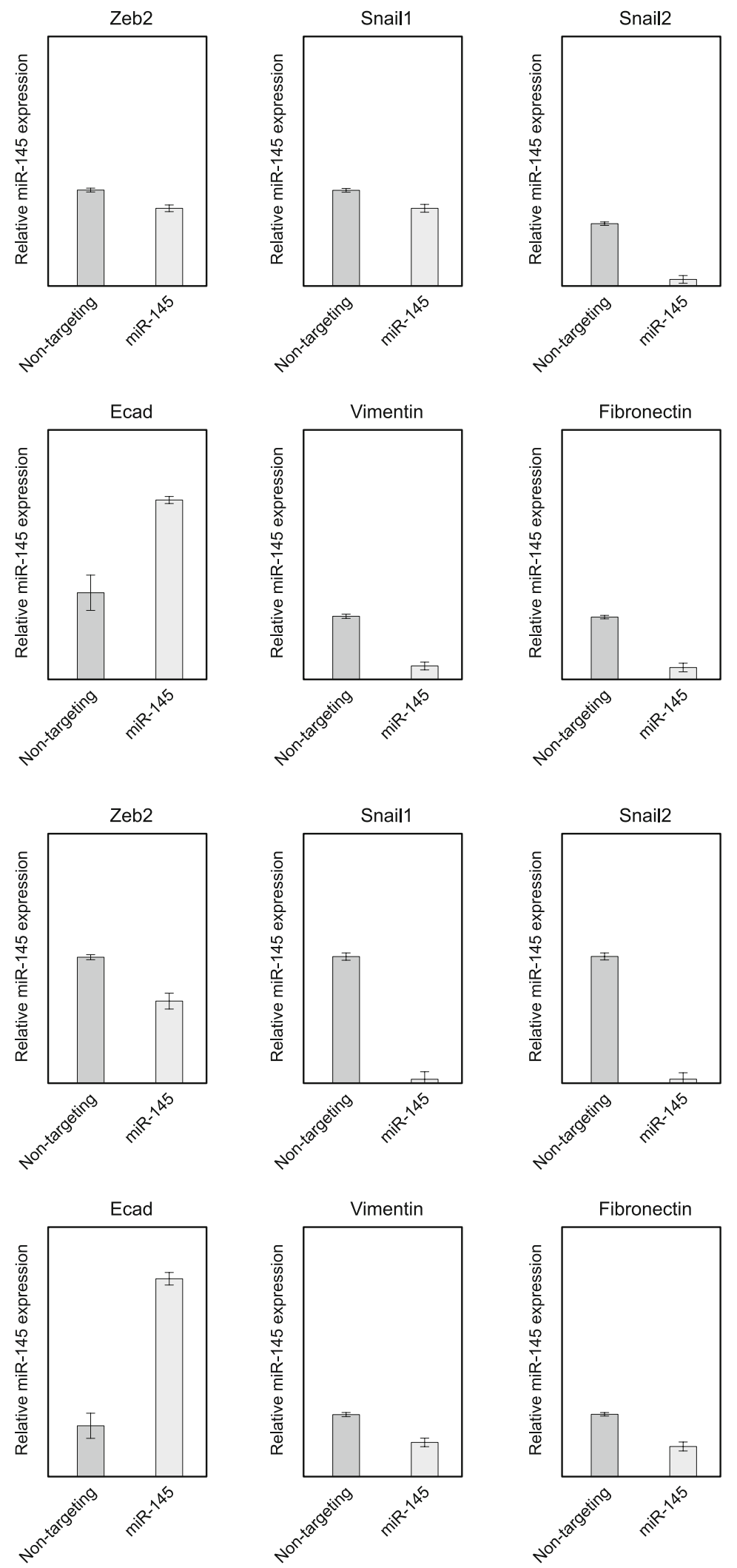

Fig. 2. CSC markers expressions are repressed by 3'UTR binding of miR-145. A) mir-145 regulated EMT through KLF4, OCT4, SOX2 and its downstream transcriptional factors, ZEB1, ZEB2, Snail1, Snail2, Vimentin, Fibronectin in TC71 and B) TC106. 
A

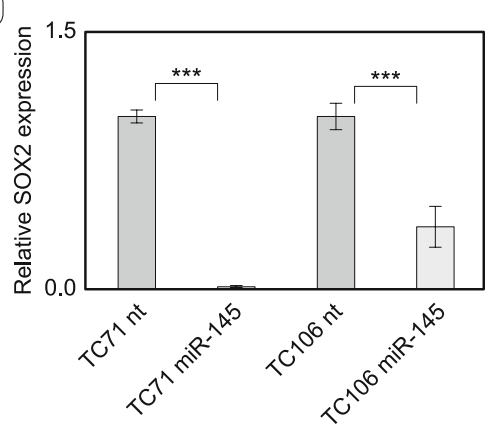

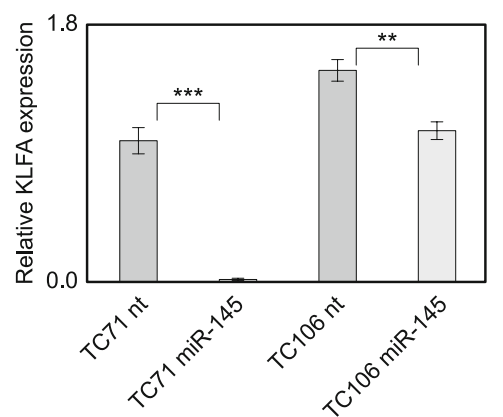
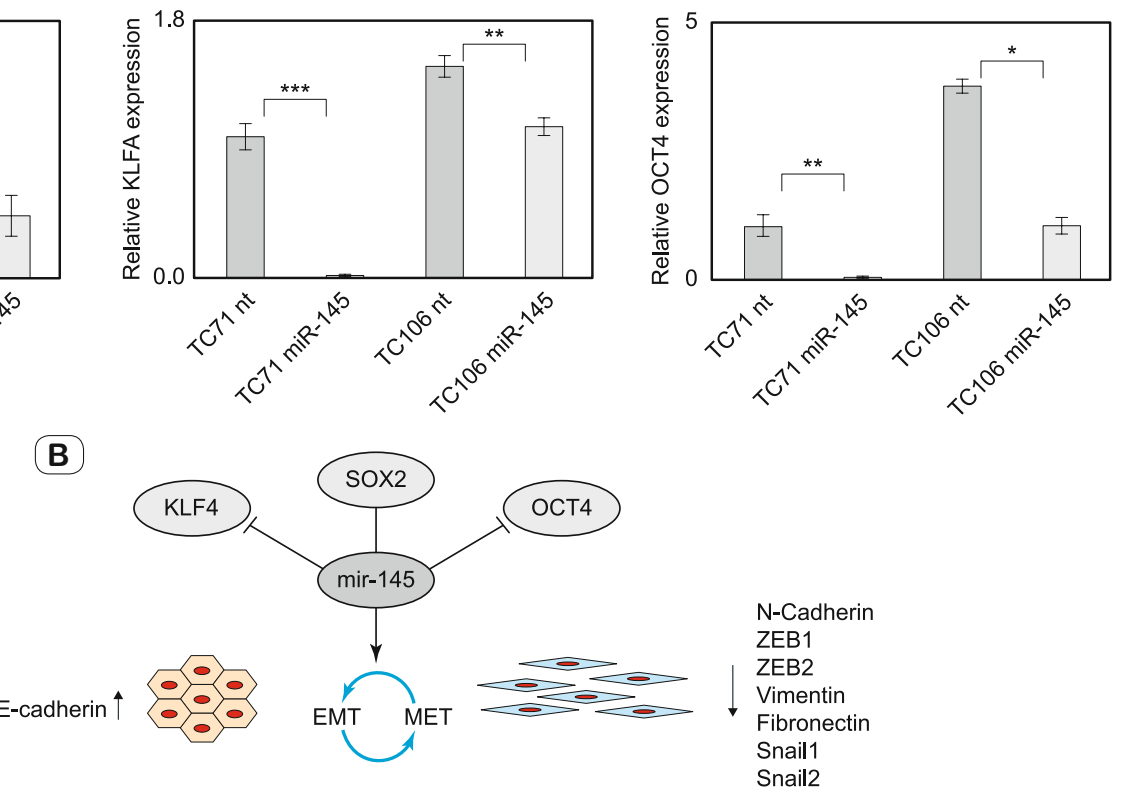

Fig. 3. (A) Treatment with miR-145 in TC71 and TC106 cells inhibited the mRNA levels of KLF4, OCT4, SOX2. Statistical analysis was performed by $\mathrm{t}$ test. * $\mathrm{p}<0.01$, significant difference between two compared groups. Error bar indicates SD. (B) mir-145 regulating EMT-CSC networks.

\section{Discussion}

This current study demonstrated the tumor suppressive behavior of miR-145 in EWS cells. miR-145 expression was found to be downregulated in EWS cell lines, and miR-145 overexpression was revealed to inhibit tumorigenesis in colorectal (14), gastric (15) breast (16), larynx (6) and prostate cancer (17). To date, this is the first paper to demonstrate the function of miR-145 in TC71 and TC106 cell lines, and our data suggest that mir-145 has a potential role in EMT in EWS progression by targeting stem cell markers. We verified EMT markers and SOX2, KLF4 and OCT4 expressions as a direct target gene of miR-145 in QRT-PCR analysis.

Mir-145 is a miRNA with a high level of conservation among species, targeting multiple oncogenes, regulating various biological alterations such as cell cycle, proliferation, and apoptosis, with many cancer types (5). There is evidence that OCT4, SOX2 and KLF4, which are among the main actors involved in reprogramming of cells, are among the genes targeted by mir-145 (18).

With functional studies, mir-145 was found to have a tumor suppressor effect in Ewing sarcoma and we have shown that it was involved in cancer progression.

In addition, miR-145 has been demonstrated to directly target 3 UTR of SOX2, KLF4 and OCT4 and altered levels of miR-145 have been related with EMT and migration and metastasis (19, 20 ). It has been suggested in previous studies that the level of mir-145 decreases in the increase of metastatic efficacy in prostate cancer and that mir- 145 causes an increase in cancer stem cell potential. At the same time, with the decrease of mir-145 level in adenocarcinoma, it has been observed that it reduces the proliferation of OCT4, which is one of the targets of this miRNA (19).
In another study, it has been shown that mir-145 has an effective role in stem cell characteristic in laryngeal squamous carcinoma as our results (8).

In this study, we propose that the expressional and functional relation between miR-145 and Ewing Sarcoma signed this hypotesis: miR-145 levels were positively correlated in EWS cell line, and miR-145 inhibited EWS cell proliferation, migration, invasion, colony capacity and apoptosis as well as metastasis, and miR-145 is a critical regulator of KLF4, SOX2, OCT4 which may serve as a biomarker of miR-145 in EWS pathogenesis.

Another study showed overexpression of mir-145 in several EWS cell lines including SKN-MC, STA-ET-1, TC252, WE68 but in this current research we have for the first time shown the mir-145 role in TC71 and TC106 cells (21).

EMT is an important process in cancer development, epithelial cells lose their adhesion capacity and transform into mesenchymal type (22). Studies have shown that EMT increases the metastasis of CSC cells, as well as EMT confers tumor cells resistance to several therapeutic agents this is another important attribute of CSCs $(23,24)$. RAS activation in PTEN-null cells induced EMT cells and influenced CSC characteristics and metastatic behaviors (23).

Our current results showed that importance of mir-145 and its target SOX2, KLF4, OCT4 expression correlated with EMT, suggesting that they contribute to carcinogenesis in Ewing sarcoma. Apparently, miR-145 is just one of the important regulator for EWS cells. Zeb1, Zeb2 Snail 1, Snail 2, E-cad, Vimentin, and Fibronectin were upregulated in EWS cells, Zeb2 and Snail1, another two important transcriptional factors in EMT, may be affected by other mechanisms for TC71. Thus, our findings suggest that miR145 plays a uniform regulatory role in EMT. Our study is impor- 
tant because of identifying a stem cell marker gene, SOX2, as a target of one of the most important tumor suppressors, miR-145, in EWS. However, stem cell markers KLF4, OCT4, SOX2, may also be related to phenotypes related with miR-145 deregulation.

\section{Conclusion}

The present study is the first paper to reveal that miR-145 may function as a tumor suppressor by targeting SOX2, KLF4, OCT4 in Ewing Sarcoma cells. miR-145 downregulation in EWS might regulate EMT markers in the tumor cells through upregulation of cancer stem cells level. Therefore, miR-145 may be a potential biomarker for EWS diagnosis and may serve as a novel target for EWS therapy.

\section{References}

1. Delattre O, Zucman J, Melot T, Garau XS, Zucker JM, Lenoir GM et al. The Ewing family of tumors - a subgroup of small-round-cell tumors defined by specific chimeric transcripts. N Engl J Med 1994; 331 (5): 294-299.

2. Nasaka S, Gundeti S, Ganta RR, Arigela RS, Linga VG, Maddali LS. Impact of treatment protocol on outcome of localized Ewing's sarcoma. South Asian J Cancer 2016; 5 (4): 194-195.

3. Guzel E, Karatas OF, Semercioz A, Ekici S, Aykan S, Yentur S et al. Identification of microRNAs differentially expressed in prostatic secretions of patients with prostate cancer. Int J Cancer 2015; 136 (4): 875-879.

4. Zhang B, Farwell MA. microRNAs: a new emerging class of players for disease diagnostics and gene therapy. J Cell Mol Med 2008; 12 (1): 3-21.

5. Cui SY, Wang R, Chen LB. MicroRNA-145: a potent tumour suppressor that regulates multiple cellular pathways. J Cell Mol Med 2014; 18 (10): 1913-1926.

6. Karatas OF, Yuceturk B, Suer I, Yilmaz M, Cansiz H, Solak M et al. Role of miR-145 in human laryngeal squamous cell carcinoma. Head Neck 2016; 38 (2): 260-266.

7. Slaby O, Svoboda M, Fabian P, Smerdova T, Knoflickova D, Bednarikova $\mathbf{M}$ et al. Altered expression of miR-21, miR-31, miR-143 and miR-145 is related to clinicopathologic features of colorectal cancer. Oncology 2007; 72 (5-6): 397-402.

8. Karatas OF, Suer I, Yuceturk B, Yilmaz M, Hajiyev Y, Creighton CJ et al. The role of miR-145 in stem cell characteristics of human laryngeal squamous cell carcinoma Hep-2 cells. Tumour Biol 2016; 37 (3): 4183-4192.

9. Starczynowski DT, Morin R, McPherson A, Lam J, Chari R, Wegrzyn $\mathbf{J}$ et al. Genome-wide identification of human microRNAs located in leukemia-associated genomic alterations. Blood 2011; 117 (2): 595-607.

10. Schaefer T, Lengerke C. SOX2 protein biochemistry in stemness, reprogramming, and cancer: the PI3K/AKT/SOX2 axis and beyond. Oncogene 2020; 39 (2): 278-292.

11. Xu N, Papagiannakopoulos T, Pan G, Thomson JA, Kosik KS. MicroRNA-145 regulates OCT4, SOX2, and KLF4 and represses pluripotency in human embryonic stem cells. Cell 2009; 137 (4): 647-658.
12. Mani SA, Guo W, Liao MJ, Eaton EN, Ayyanan A, Zhou AY et al. The epithelial-mesenchymal transition generates cells with properties of stem cells. Cell 2008; 133 (4): 704-715.

13. Zaravinos A. The Regulatory Role of MicroRNAs in EMT and Cancer. J Oncol 2015; 2015: 865816.

14. Bandrés E, Cubedo E, Agirre X, Malumbres R, Zárate R, Ramirez $\mathbf{N}$ et al. Identification by Real-time PCR of 13 mature microRNAs differentially expressed in colorectal cancer and non-tumoral tissues. Mol Cancer 2006; 5: 29.

15. Takagi T, Iio A, Nakagawa $Y$, Naoe T, Tanigawa N, Akao Y. Decreased expression of microRNA-143 and -145 in human gastric cancers. Oncology 2009; 77 (1): 12-21.

16. Sempere LF, Christensen M, Silahtaroglu A, Bak M, Heath CV, Schwartz G et al. Altered MicroRNA expression confined to specific epithelial cell subpopulations in breast cancer. Cancer Res 2007; 67 (24): 11612-11620.

17. Ozen M, Karatas OF, Gulluoglu S, Bayrak OF, Sevli S, Guzel E et al. Overexpression of miR-145-5p inhibits proliferation of prostate cancer cells and reduces SOX2 expression. Cancer Invest 2015; 33 (6): 251-258.

18. Pashaei E, Guzel E, Ozgurses ME, Demirel G, Aydin N, Ozen M. A Meta-Analysis: Identification of Common MiR-145 Target Genes that have Similar Behavior in Different GEO Datasets. PLoS One 2016; 11 (9): e0161491.

19. Hu J, Qiu M, Jiang F, Zhang S, Yang X, Wang J et al. MiR-145 regulates cancer stem-like properties and epithelial-to-mesenchymal transition in lung adenocarcinoma-initiating cells. Tumour Biol 2014; 35 (9): 8953-8961.

20. Noguchi S, Yasui Y, Iwasaki J, Kumazaki M, Yamada N, Naito S et al. Replacement treatment with microRNA-143 and -145 induces synergistic inhibition of the growth of human bladder cancer cells by regulating PI3K/Akt and MAPK signaling pathways. Cancer Lett 2013; 328 (2): 353-361.

21. Ban J, Jug G, Mestdagh P, Schwentner R, Kauer M, Aryee DN et al. Has-miR-145 is the top EWS-FLI1-repressed microRNA involved in a positive feedback loop in Ewing's sarcoma. Oncogene 2011; 30 (18): 2173-2180.

22. Lee JM, Dedhar S, Kalluri R, Thompson EW. The epithelial-mesenchymal transition: new insights in signaling, development, and disease. J Cell Biol 2006; 172 (7): 973-981.

23. Mulholland DJ, Kobayashi N, Ruscetti M, Zhi A, Tran LM, Huang $\mathbf{J}$ et al. Pten loss and RAS/MAPK activation cooperate to promote EMT and metastasis initiated from prostate cancer stem/progenitor cells. Cancer Res 2012; 72 (7): 1878-1889.

24. Klarmann GJ, Hurt EM, Mathews LA, Zhang X, Duhagon MA, Mistree $\mathbf{T}$ et al. Invasive prostate cancer cells are tumor initiating cells that have a stem cell-like genomic signature. Clin Exp Metastasis 2009; 26 (5): 433-446.

25. Suer I, Karatas OF, Yuceturk B, Yilmaz M, Guven G, Buge O et al. Characterization of stem-like cells directly isolated from freshly resected laryngeal squamous cell carcinoma specimens. Curr Stem Cell Res Ther 2014; 9 (4): 347-353.

26. Casas E, Kim J, Bendesky A, Ohno-Machado L, Wolfe CJ, Yang J. Snail2 is an essential mediator of Twist1-induced epithelial mesenchymal transition and metastasis. Cancer Res 2011; 71 (1): 245-254.

Received July 15, 2020. Accepted September 20, 2020. 